\title{
What distinguishes unintentional injuries from injuries due to intimate partner violence: a study in Greek ambulatory care settings
}

\author{
E Petridou, A Browne, E Lichter, X Dedoukou, D Alexe, N Dessypris
}

Injury Prevention 2002;8:197-201

See end of article for authors' affiliations

\section{Correspondence to:}

Dr Eleni Petridou, Associate

Professor of Epidemiology,

Department of

Epidemiology, Athens

University Medical School,

75 Mikras Asias Str,

Athens 11527, Greece;

epetrid@med.voa.gr

\begin{abstract}
Objectives: Intimate partner violence (IPV) is an important sociocultural and public health problem. This study aims to assess sociodemographic and injury characteristics of IPV victims among adults in a traditional southern European population.

Setting: Accident and emergency departments of three sentinel hospitals in Greece participating in the Emergency Department Injury Surveillance System (EDISS).

Methods: Data on sociodemographic variables, as well as event and injury characteristics were retrieved from the EDISS database during the three year period 1996-98. Out of a total of 27319 injured women aged 19 years or more, $312(1.1 \%)$ were reported as IPV related and were compared with 26466 women with unintentional injuries. Among the 35174 men with injuries $39(0.1 \%)$ were reported as IPV related and were compared with 34049 men with unintentional injuries. The data were analyzed through simple cross tabulations and multiple logistic regression. Positive predicted values for selected injury characteristics were also calculated.

Results: IPV is more common in rural than in urban areas of Greece. Women are 10 times more frequently IPV victims but men are also IPV victims; younger women and older men are disproportionately affected by IPV. The relative frequency of the phenomenon increases during the late evening and night hours. Certain types of injuries, notably multiple facial injuries, and presentation of the injured person on his/her own at the emergency department or combinations of predictive characteristics are strongly indicative of IPV.

Conclusions: Injuries due to IPV are not uncommon in Greece, not withstanding the traditional structure of the society and the tendency of under-reporting. Certain injury characteristics have high positive predictive values and could be used in screening protocols aiming at the correct identification of the underlying external cause in injuries that may be caused by IPV.
\end{abstract}

V iolence between intimate partners is an important but underdiagnosed sociocultural, legal, and public health problem. Intimate partner violence (IPV)—defined here as physical aggression between couples in marital or intimate relationships-affects adults of all ages, nationalities, and socioeconomic levels. IPV has a short and long term impact on the physical, emotional, and social wellbeing of victims and perpetrators, even if the violence is hidden from the rest of the world.

Detection and appropriate care for injuries due to IPV is critical for the long term physical and mental health of patients. Health professionals, especially those involved in primary health care, have the opportunity to act as crucial links between persons who experience IPV and medical and community based resources. However, there is evidence that diagnosis of IPV in primary health care settings is quite low compared with its frequency. ${ }^{2}{ }^{3}$ Patients' disclosure rates in medical settings are not representative of the magnitude of the problem. The shortage of time to provide care, combined with health care providers' reluctance to investigate family issues in patients' lives, also contributes to the lack of accurate documentation of intimate partner abuse. ${ }^{4}$ Skill training for health professionals, supported by implementation of focused screening tools for the identification of cases, may be a promising preventive strategy.

In recent years, new awareness of the health consequences of partner violence has resulted in an increased emphasis by health care providers in some countries on identifying injuries resulting from such violence. The authors of this article recognize the need for a similar effort in Greece and other countries in the European Union. In Greece, although there is some evidence that violent family relationships exist, they are not a topic of public discussion and protocols designed to identify IPV in health care settings have not been developed. The study reported here is an attempt to describe cases presenting with injuries reported as due to partner violence in urban and rural health settings.

The goal of these analyses is to identify sociodemographic and injury characteristics of individuals presenting to accident and emergency departments in Greece as victims of physical IPV. The study is based on data from the Emergency Department Injury Surveillance System (EDISS). ${ }^{5}$ The immediate objectives are to calculate positive predictive values of particular manifestations and identify characteristics with high predictive values as markers indicating likely partner abuse.

\section{METHODS}

Established in 1986 by the European Union, the European Home and Leisure Accident Surveillance System (EHLASS) collects data on injuries diagnosed in accident and emergency departments in hospitals. At present, 13 out of 15 member states of the European Union participate in EHLASS, with some variations in data collection methods, whereas the other two countries use data from household surveys. In Greece the EDISS is run by the Greek Center for Research and Prevention of Injuries among the Young and collects data on all ages and

Abbreviations: $\mathrm{Cl}$, confidence interval; EDISS, Emergency Department Injury Surveillance System; EHLASS, European Home and Leisure Accident Surveillance System; IPV, intimate partner violence; OR, odds ratio 
all types of injuries. Four hospitals were chosen as EDISS data collection sites. Two of them are located in the Greater Athens area; one is a major trauma hospital and the other is a children's teaching hospital, which obviously did not contribute data for the present analyses. Injury data representing the rural areas of the country are collected from two district hospitals, one on the island of Corfu and the other in Magnesia County on the mainland. All participating hospitals have residency programs. The Trauma Hospital in Athens, being one of the two major trauma hospitals in the Greater Athens area, is likely to capture a representative sample of injury cases. With respect to the rural areas, a validation against the National Statistical Service of Greece hospital discharge trauma data did not indicate any sharp deviation from representativeness.

Analyses for this study were based on injury data from women and men over 19 years of age, collected during a three year period from 1996-98. Specially trained health visitors, who are equivalent to registered nurses in the United States, conducted in-person interviews with all individuals who presented at accident and emergency departments of the Trauma Hospital in Athens and the two district hospitals, using a standardized questionnaire. All 62493 cases of intentional and unintentional injuries, who contacted the emergency departments of the participating hospitals during the study period, were interviewed in person by our trained health visitors in the presence of the attending physician. Each interview lasted for about seven minutes, but when IPV was suspected, the interviewee was encouraged to discuss freely her/his problem. The questions probed IPV by focusing on the mechanism of injury and in particular, whether another person was involved in the causation of injury. We did not use established IPV screening instruments because the objective of the study was to explore the potential of an existing injury surveillance system to identify and assess the dimensions of the IPV issue before undertaking more specialized studies. Therefore, we do not claim that our study meets the requirements of a formal IPV screening program. Fewer than $1 \%$ of all eligible persons were not interviewed in the emergency department because of technical or administrative problems and none of the injured persons refused to collaborate, because EDISS is integrated in the routine functions of the emergency departments of the participating hospitals. We have avoided the use of proxy responders, except in rare circumstances (less than $2 \%$ ), of mainly critically ill patients, who, in any case, are also interviewed in the wards. Information on sociodemographic features (gender, age, nationality), event characteristics (place, conditions, time), and nature of injury (type of injury, injured body part, number of injuries) were gathered. Three categories of injuries were subsequently created: (1) physical injuries reported to have been inflicted by an intimate partner, irrespective of current cohabitation with the perpetrator; (2) injuries due to all other types of interpersonal violence; and (3) unintentional injuries.

The statistical analyses were conducted using the SAS program. Preliminary analyses involved simple cross tabulations. Subsequently, the data were modeled through multiple logistic regression in order to control for mutual confounding among variables routinely collected in this surveillance system and to calculate adjusted odds ratios for a variety of characteristics. Type of injury and injured body part were alternatively introduced in the core model to avoid colinearity problems between these two variables. The decision was made to compare intimate partner related injuries with unintentional injuries, because intentional injuries have their own specific manifestation pattern. Thus, odds ratios reflect the likelihood that an injury was due to intimate partner violence rather than an unintentional cause. Separate analyses by gender were conducted because the epidemiological pattern of intimate partner injuries among women is very different from that among men. Findings were considered significant at $\mathrm{p}<0.05$. Finally, positive predictive values for IPV were calculated for injuries of specific characteristics. Positive predictive values are calculated by dividing the number of IPV injuries of certain characteristic(s) by the total number of injuries of the same characteristics (the total includes IPV injuries and unintentional injuries). Characteristics associated with high predictive values can be used as indicators of IPV, even when such information has not been forthcoming from the injured person.

\section{RESULTS}

During the three year period of the study, 62493 cases of intentional and unintentional injuries were recorded. Of these, 312 among women (1.1\% of injured women) and 39 among men $(0.1 \%$ of injured men) were reported as caused by IPV. During the same period 26466 women and 34049 men reported injuries attributed to unintentional causes, whereas 541 women $(2.0 \%$ of injured women) and 1086 men $(3.1 \%$ of injured men) reported injuries due to violence inflicted by individuals other than their intimate partners. Table 1 (women) and table 2 (men) provide frequency distributions of persons with injuries caused by IPV and persons with unintentional injuries by sociodemographic variables, time and place of the injury causing event, and injury characteristics. These tables provide a descriptive overview, but do not reveal the independent association of IPV with injuries of specific characteristics, because of mutual confounding among the studied variables. Mutually adjusted findings for women and men are presented in tables 3 and 4, respectively.

\section{Findings related to women (table 1)}

Nearly half of the women reporting an injury due to IPV were aged 30-39 and over one fifth were less than 30 years of age. The large majority of injuries to women identified as resulting from IPV occurred at home (87\%). Over half occurred between the hours of $2 \mathrm{pm}$ and $10 \mathrm{pm}$ (54\%) and nearly three quarters were inflicted on a weekday (72\%). Most of partner violence injuries identified in women were to the head and face $(62 \%)$ and most presented at the hospital with multiple injuries $(60 \%)$. The majority of women's injuries due to IPV were contusions or open wounds (72\%), whereas nearly one fifth (18\%) of injuries were concussions; there were $4 \%$ fractures and another 5\% were dislocations.

\section{Findings related to men (table 2)}

Nearly one third of men who reported injuries by intimate partners were in their 30s (31\%), 36\% were aged 40-60, and only $10 \%$ were under the age of 30 . As among women, the large majority of men's IPV injuries also occurred at home $(90 \%)$. Over half of male IPV injuries occurred between the hours of $2 \mathrm{pm}$ and $10 \mathrm{pm}$ (59\%) and over half were located in the head and face $(62 \%)$. Injuries sustained by men consisted mostly of contusions ( $87 \%$ ) and no man suffered a fracture or dislocation because of IPV.

\section{Comparisons between IPV and unintentional injuries}

Table 3 (women) and table 4 (men) provide multiple logistic regression derived adjusted odds ratios (ORs) and 95\% confidence intervals (CIs).

\section{Findings related to women (table 3 )}

Injuries reported by women aged 30-39 were more than twice as likely to be due to IPV than to unintentional injury, when compared with the reference category of women aged 40-49 (OR 2.32; 95\% CI 1.64 to 3.27). The likelihood that an injury was due to intimate partner violence declined sharply after the age of 50. IPV was more than four times as likely to be reported as the cause of injury by women in rural areas as by women in urban areas (OR 4.35; 95\% CI 3.00 to 6.32). However, Greek women were not more likely than women of other nationalities living in Greece to report IPV related injuries.

In reports by women, day of the week and season of the year were not significantly related to injuries resulting from IPV, although the likelihood of injury due to IPV was higher during 


\begin{tabular}{|c|c|c|}
\hline Variable & $\begin{array}{l}\text { No (\%) of } \\
\text { injuries due } \\
\text { to IPV }\end{array}$ & $\begin{array}{l}\text { No }(\%) \text { of } \\
\text { injuries declarec } \\
\text { as unintentiona }\end{array}$ \\
\hline \multicolumn{3}{|l|}{ Age (years) } \\
\hline$<30$ & $73(23.4)$ & $4965(18.8)$ \\
\hline $30-39$ & $136(43.6)$ & 3703 (14.0) \\
\hline $40-49$ & $60(19.2)$ & 3579 (13.5) \\
\hline $50-59$ & $28(9.0)$ & 3825 (14.4) \\
\hline $60+$ & $15(4.8)$ & $10394(39.3)$ \\
\hline \multicolumn{3}{|l|}{ Nationality } \\
\hline Greek & $287(92.0)$ & $25032(94.6)$ \\
\hline Migrants & $25(8.0)$ & $1434(5.4)$ \\
\hline \multicolumn{3}{|l|}{ Region } \\
\hline Urban & $37(11.9)$ & $10809(40.8)$ \\
\hline Rural & $275(88.1)$ & 15657 (59.2) \\
\hline \multicolumn{3}{|l|}{ Place } \\
\hline Home & $272(87.2)$ & $13407(50.7)$ \\
\hline Other & $40(12.8)$ & $13059(49.3)$ \\
\hline \multicolumn{3}{|l|}{ Time } \\
\hline $24.00-05.59$ & $21(6.7)$ & $788(3.0)$ \\
\hline $06.00-13.59$ & 89 (28.5) & $12787(48.3)$ \\
\hline $14.00-21.59$ & $167(53.5)$ & $11327(42.8)$ \\
\hline $22.00-23.59$ & 35 (11.3) & $1564(5.9)$ \\
\hline \multicolumn{3}{|l|}{ Day } \\
\hline Weekday & $226(72.4)$ & $19370(73.2)$ \\
\hline Weekend & $86(27.6)$ & $7096(26.8)$ \\
\hline \multicolumn{3}{|l|}{ Season } \\
\hline Winter & 62 (19.9) & $5827(22.0)$ \\
\hline Spring & 68 (21.8) & $6561(24.8)$ \\
\hline Summer & $104(33.3)$ & $7289(27.5)$ \\
\hline Autumn & $78(25.0)$ & $6789(25.7)$ \\
\hline \multicolumn{3}{|l|}{ Transfer of injured person } \\
\hline On her own & $124(39.7)$ & $2353(8.9)$ \\
\hline Ambulance/other person & $188(60.3)$ & $24113(91.1)$ \\
\hline \multicolumn{3}{|c|}{ Time from injury to medical evaluation } \\
\hline Same day & $228(73.1)$ & $20769(78.5)$ \\
\hline More than one day & $84(26.9)$ & $5697(21.5)$ \\
\hline \multicolumn{3}{|l|}{ Number of injuries } \\
\hline One & $124(39.7)$ & $21757(82.2)$ \\
\hline Multiple & $188(60.3)$ & $4709(17.8)$ \\
\hline \multicolumn{3}{|l|}{ Type of injury } \\
\hline Contusion/open wound & $223(71.5)$ & $12904(48.8)$ \\
\hline Fracture & $12(3.9)$ & $7047(26.6)$ \\
\hline Concussion & 55 (17.6) & $1408(5.3)$ \\
\hline Luxation/dislocation/other & $16(5.1)$ & $4814(18.2)$ \\
\hline No injury diagnosed & $6(1.9)$ & $290(1.1)$ \\
\hline \multicolumn{3}{|l|}{ Body part inured } \\
\hline Brain & $56(17.9)$ & $1441(5.4)$ \\
\hline Skull & 43 (13.8) & $1007(3.8)$ \\
\hline Face/rest of the head & $94(30.1)$ & $1271(4.8)$ \\
\hline Trunk & 51 (16.4) & $3836(14.5)$ \\
\hline Upper limbs & $52(16.7)$ & $8182(30.9)$ \\
\hline Lower limbs & $16(5.1)$ & $10729(40.6)$ \\
\hline
\end{tabular}

the night hours than during the day hours. Women who came to the emergency medical departments alone were more than four times as likely as women transferred to emergency departments by ambulance or brought by another person to report that their injuries were due to IPV (OR 4.75; 95\% CI 3.59 to 6.28 ). Women with multiple injuries were 15 times more likely to report IPV as the cause of their injury as were women who presented at the emergency departments with a single injury (OR 15.15; 95\% CI 11.61 to 19.77). Injuries caused by IPV were, as indicated, concentrated in the brain, skull, and facial region. The odds that injuries in these regions (compared with the reference category, upper and lower limbs) were due to IPV rather than unintentional cause exceeded the value of 13 .

\section{Findings related to men (table 4)}

In contrast to women, age was not significantly related to IPV injuries among men, and injuries due to IPV among men were
Table 2 Distribution of the 34088 men aged over 19 , with injuries recorded in EDISS during a three year period (1996-98) by demographic, event and injury descriptive variables, and by the cause of injury

\begin{tabular}{|c|c|c|}
\hline Variable & $\begin{array}{l}\text { No (\%) of } \\
\text { injuries due } \\
\text { to IPV }\end{array}$ & $\begin{array}{l}\text { No }(\%) \text { of } \\
\text { injuries declared } \\
\text { as unintentional }\end{array}$ \\
\hline \multicolumn{3}{|l|}{ Age (years) } \\
\hline$<30$ & $4(10.3)$ & $11295(33.2)$ \\
\hline $30-39$ & $12(30.8)$ & $7261(21.3)$ \\
\hline $40-49$ & 7 (17.9) & $5387(15.8)$ \\
\hline $50-59$ & 7 (17.9) & $3969(11.7)$ \\
\hline $60+$ & $9(23.1)$ & $6137(18.0)$ \\
\hline \multicolumn{3}{|l|}{ Nationality } \\
\hline Greek & $37(94.9)$ & $31344(92.1)$ \\
\hline Migrants & $2(5.1)$ & $2705(7.9)$ \\
\hline \multicolumn{3}{|l|}{ Region } \\
\hline Urban & $4(10.3)$ & $11311(33.2)$ \\
\hline Rural & $35(89.7)$ & $22738(66.8)$ \\
\hline \multicolumn{3}{|l|}{ Place } \\
\hline Home & $35(89.7)$ & $8129(23.9)$ \\
\hline Other & $4(10.3)$ & 25920 (76.1) \\
\hline \multicolumn{3}{|l|}{ Time } \\
\hline $24.00-05.59$ & $3(7.7)$ & $1267(3.7)$ \\
\hline $06.00-13.59$ & $10(25.6)$ & $15059(44.2)$ \\
\hline $14.00-21.59$ & $23(59.0)$ & $15440(45.4)$ \\
\hline $22.00-23.59$ & $3(7.7)$ & $2283(6.7)$ \\
\hline \multicolumn{3}{|l|}{ Day } \\
\hline Weekday & $22(56.4)$ & $24664(72.4)$ \\
\hline Weekend & $17(43.6)$ & $9385(27.6)$ \\
\hline \multicolumn{3}{|l|}{ Season } \\
\hline Winter & $6(15.4)$ & $7297(21.4)$ \\
\hline Spring & $6(15.4)$ & $8133(24.0)$ \\
\hline Summer & $14(35.9)$ & $9533(28.0)$ \\
\hline Autumn & $13(33.3)$ & $9036(26.6)$ \\
\hline \multicolumn{3}{|l|}{ Transfer of injured person } \\
\hline On his own & $19(48.7)$ & $8399(24.7)$ \\
\hline Ambulance/other person & $20(51.3)$ & $25650(75.3)$ \\
\hline \multicolumn{3}{|c|}{ Time from injury to medical evaluation } \\
\hline Same day & $33(84.6)$ & $27694(81.3)$ \\
\hline More than one day & $6(15.4)$ & 6355 (18.7) \\
\hline \multicolumn{3}{|l|}{ Number of injuries } \\
\hline One & $19(48.7)$ & $26742(78.5)$ \\
\hline Multiple & $20(51.3)$ & $7307(21.5)$ \\
\hline \multicolumn{3}{|l|}{ Type of injury } \\
\hline Contusion/open wound & $34(87.2)$ & $11299(59.6)$ \\
\hline Fracture & $0(0.0)$ & $6536(16.6)$ \\
\hline Concussion & $3(7.6)$ & $1855(5.4)$ \\
\hline Luxation/dislocation/other & $1(2.6)$ & $5969(17.5)$ \\
\hline No injury diagnosed & $1(2.6)$ & $290(0.9)$ \\
\hline \multicolumn{3}{|l|}{ Body part injured } \\
\hline Brain & $3(7.7)$ & $1889(5.5)$ \\
\hline Skull & 7 (17.9) & $1658(4.9)$ \\
\hline Face/rest of the head & $14(35.9)$ & $3362(9.9)$ \\
\hline Trunk & $8(20.5)$ & 4635 (13.6) \\
\hline Upper limbs & $6(15.4)$ & $11512(33.8)$ \\
\hline Lower limbs & $1(2.6)$ & $10993(32.3)$ \\
\hline
\end{tabular}

generally less serious than unintentional injuries. As with women, men were almost five times more likely to suffer IPV injuries in rural as in urban areas (OR 4.99; 95\% CI 1.70 to 14.67), but ethnicity was not significantly related to intimate partner injuries. Among men who identified themselves as victims of IPV, there was a trend toward injuries occurring during weekends, rather than weekdays $(p=0.06)$. As with women, injuries reported as resulting from IPV were concentrated during the night hours. Like women, IPV related injuries were more than four times more likely to be reported by men who came to the emergency department alone rather than by ambulance or with another person (OR 3.92; 95\% CI 1.96 to 7.85 ) and more than 11 times more likely among men with multiple injuries (OR 11.75; 95\% CI 5.88 to 23.48).

Certain values of individual variables can be used to generate acceptable predictive values. For instance, among women, contusion and swelling of the lips had a predictive value in this 
Table 3 Multiple logistic regression derived odd ratios (ORs) and $95 \%$ confidence intervals $(95 \% \mathrm{Cls})$ for a woman to sustain an injury due to IPV rather than to declare an unintentional injury by a series of demographic, event and injury variables, controlling for place of occurrence of the injury

\begin{tabular}{|c|c|c|c|}
\hline Variable & ORs & $95 \% \mathrm{Cls}$ & $\mathrm{p}$ Value \\
\hline \multicolumn{4}{|l|}{ Age (years) } \\
\hline$<30$ & 1.11 & 0.76 to 1.61 & 0.60 \\
\hline $30-39$ & 2.32 & 1.64 to 3.27 & 0.0001 \\
\hline $40-49$ & Baseline & & \\
\hline $50-59$ & 0.43 & 0.27 to 0.69 & 0.0005 \\
\hline $60+$ & 0.07 & 0.04 to 0.13 & 0.0001 \\
\hline \multicolumn{4}{|l|}{ Nationality } \\
\hline Greek & Baseline & & \\
\hline Migrants & 1.40 & 0.80 to 2.47 & 0.24 \\
\hline \multicolumn{4}{|l|}{ Region } \\
\hline Urban & Baseline & & \\
\hline Rural & 4.35 & 3.00 to 6.32 & 0.0001 \\
\hline \multicolumn{4}{|l|}{ Time } \\
\hline $24.00-05.59$ & 2.76 & 1.55 to 4.92 & 0.0006 \\
\hline $06.00-13.59$ & Baseline & & \\
\hline $14.00-21.59$ & 1.35 & 1.02 to 1.81 & 0.04 \\
\hline $22.00-23.59$ & 2.26 & 1.41 to 3.63 & 0.0007 \\
\hline \multicolumn{4}{|l|}{ Day } \\
\hline $\begin{array}{l}\text { Weekday } \\
\text { Weekend }\end{array}$ & $\begin{array}{l}1.08 \\
\text { Baseline }\end{array}$ & 0.82 to 1.43 & 0.57 \\
\hline \multicolumn{4}{|l|}{ Season } \\
\hline Winter & 0.93 & 0.64 to 1.37 & 0.72 \\
\hline Spring & Baseline & & \\
\hline Summer & 1.23 & 0.87 to 1.73 & 0.25 \\
\hline Autumn & 1.02 & 0.71 to 1.46 & 0.92 \\
\hline \multicolumn{4}{|l|}{ Transfer of injured person } \\
\hline On her own & 4.75 & 3.59 to 6.28 & 0.0001 \\
\hline Ambulance/other person & Baseline & & \\
\hline \multicolumn{4}{|c|}{ Time from injury to medical evaluation } \\
\hline Same day & Baseline & & \\
\hline More than one day & 1.22 & 0.89 to 1.68 & 0.21 \\
\hline \multicolumn{4}{|l|}{ Number of injuries } \\
\hline One & Baseline & & \\
\hline Multiple & 15.15 & 11.61 to 19.77 & 0.0001 \\
\hline \multicolumn{4}{|c|}{ Additional, alternatively introduced variables } \\
\hline \multicolumn{4}{|l|}{ Type of injury } \\
\hline Contusion/open wound & Baseline & & \\
\hline Fracture & 0.21 & 0.12 to 0.39 & 0.0001 \\
\hline Concussion & 2.55 & 1.75 to 3.71 & 0.0001 \\
\hline Luxation/dislocation & 0.08 & 0.04 to 0.18 & 0.0001 \\
\hline No injury diagnosed & 0.61 & 0.24 to 1.56 & 0.30 \\
\hline \multicolumn{4}{|l|}{ Injured body part } \\
\hline Brain & 13.21 & 8.57 to 20.38 & 0.0001 \\
\hline Skull & 12.67 & 8.05 to 19.96 & 0.0001 \\
\hline Face/rest of the head & 13.67 & 9.31 to 20.07 & 0.0001 \\
\hline Trunk & 3.49 & 2.33 to 5.21 & 0.0001 \\
\hline Upper and lower limbs & Baseline & & \\
\hline
\end{tabular}

study of about $43 \%$, meaning that a women with an unknown cause of contusion and swelling of the lips has a $43 \%$ probability to have sustained that injury because of intimate partner assault. Moreover, with a combination of predictive characteristics-for example, an unaccompanied woman 30-39 years old, from a rural area, presenting at night, with multiple injuries including the face-the injury is more likely than not (predictive value far in excess of $50 \%$ ) to have been caused as a result of IPV.

\section{DISCUSSION}

IPV is a universal phenomenon and-however underreported-it has been documented in all populations that have been properly investigated. ${ }^{6-8}$ This investigation shows that IPV also exists in Greece and is more common in rural areas. Women are far more frequently IPV victims but men are not immune to this phenomenon. IPV can occur whenever the
Table 4 Multiple logistic regression derived odd ratios (ORs) and $95 \%$ confidence intervals $(95 \% \mathrm{Cls})$ for a man to sustain an injury due to IPV rather than other causes of injuries except from violence, by a series of demographic, accident and injury variables, controlling for place of occurrence of the injury

\begin{tabular}{|c|c|c|c|}
\hline Variable & ORs & $95 \% \mathrm{Cls}$ & $\mathrm{p}$ Value \\
\hline \multicolumn{4}{|l|}{ Age (years) } \\
\hline$<30$ & 0.44 & 0.13 to 1.52 & 0.19 \\
\hline $30-39$ & 1.44 & 0.56 to 3.72 & 0.45 \\
\hline $40-49$ & Baseline & & \\
\hline $50-59$ & 1.28 & 0.44 to 3.70 & 0.65 \\
\hline $60+$ & 0.77 & 0.27 to 2.16 & 0.62 \\
\hline \multicolumn{4}{|l|}{ Nationality } \\
\hline Greek & Baseline & & \\
\hline Migrants & 1.59 & 0.35 to 7.15 & 0.54 \\
\hline \multicolumn{4}{|l|}{ Region } \\
\hline Urban & Baseline & & \\
\hline Rural & 4.99 & 1.70 to 14.67 & 0.003 \\
\hline \multicolumn{4}{|l|}{ Time } \\
\hline $24.00-05.59$ & 3.87 & 1.01 to 14.89 & 0.05 \\
\hline $06.00-13.59$ & Baseline & & \\
\hline $14.00-21.59$ & 1.79 & 0.83 to 3.85 & 0.14 \\
\hline $22.00-23.59$ & 3.39 & 0.85 to 13.49 & 0.08 \\
\hline \multicolumn{4}{|l|}{ Day } \\
\hline Weekday & 0.54 & 0.28 to 1.03 & 0.06 \\
\hline Weekend & Baseline & & \\
\hline \multicolumn{4}{|l|}{ Season } \\
\hline Winter & 1.29 & 0.41 to 4.06 & 0.66 \\
\hline Spring & Baseline & & \\
\hline Summer & 2.31 & 0.87 to 6.13 & 0.09 \\
\hline Autumn & 2.45 & 0.91 to 6.58 & 0.07 \\
\hline \multicolumn{4}{|l|}{ Transfer of injured person } \\
\hline On his own & 3.92 & 1.96 to 7.85 & 0.0001 \\
\hline Ambulance/other person & Baseline & & \\
\hline \multicolumn{4}{|c|}{ Time from injury to medical evaluation } \\
\hline Same day & Baseline & & \\
\hline More than one day & 0.54 & 0.21 to 1.41 & 0.21 \\
\hline \multicolumn{4}{|l|}{ Number of injuries } \\
\hline One & Baseline & & \\
\hline Multiple & 11.75 & 5.88 to 23.48 & 0.0001 \\
\hline \multicolumn{4}{|c|}{ Additional, alternatively introduced variables } \\
\hline \multicolumn{4}{|l|}{ Type of injury } \\
\hline Contusion/open wound & Baseline & & \\
\hline Fracture/concussion & 0.23 & 0.07 to 0.76 & 0.02 \\
\hline Luxation/dislocation & 0.10 & 0.01 to 0.75 & 0.02 \\
\hline No injury diagnosed & 2.85 & 0.37 to 21.96 & 0.31 \\
\hline \multicolumn{4}{|l|}{ Injured body part } \\
\hline Brain & 5.46 & 1.29 to 23.09 & 0.02 \\
\hline Skull & 14.37 & 4.71 to 43.83 & 0.0001 \\
\hline Face/rest of the head & 11.31 & 4.36 to 29.36 & 0.0001 \\
\hline Trunk & 5.35 & 1.89 to 15.15 & 0.002 \\
\hline Upper and lower limbs & Baseline & & \\
\hline
\end{tabular}

partners coexist, but the relative frequency of the phenomenon increases during the late evening and night hours, possibly because alcohol intoxication plays an interactive part, although the latter hypothesis could not be assessed in this surveillance system. Younger women and older men are disproportionally affected by IPV. Certain types of injuries, notably multiple facial injuries, and certain modes of presentation at the emergency department, notably presentation of the injured person on his/her own, are strongly indicative of IPV. This is a finding of some importance, because IPV victims tend to grossly under-report their injuries, especially in traditional societies.

Overall, the frequency of IPV identified in this study is low, compared with that in other countries. For example, between $2 \%$ and $12 \%$ of women's acute injuries seen in emergency departments in the United States are due to IPV, ${ }^{2}{ }^{10}$ although this wide range may, at least in part, illustrate the difficulty in interpreting IPV definitions across studies. To a certain extent, 
the lower proportion of IPV found in the Greek study may be due to the lack of focused screening protocols for IPV. Another probability is reluctance among patients to report IPV in a culture that views violence within families as occurring only infrequently in that society.

Underdiagnosis, however, is a serious problem across all national settings. Recent studies in North America suggest that fewer than half of those who present with injuries due to partner violence are identified as victims of intimate partners. ${ }^{3}$ In other studies, however, only $7 \%$ to $25 \%$ of injuries inflicted by a partner are identified as such. ${ }^{2}$ An unknown fraction of IPV injuries may have been missed in the Greek medical settings as well.

There are many barriers to identification of IPV in health care settings. Health care providers often fail to connect a patient's injuries with IPV, and are hesitant to discuss family issues with their patients because of a lack of training and education on risk factors and prevalence of the phenomenon. Similarly, physicians may not feel comfortable intervening if a patient discloses family violence, or may fear offending the patient by asking about interpersonal family relationships. ${ }^{11}{ }^{12}$ Research outside Greece indicates that time constraints also limit a physician's likelihood of asking about violence within families. ${ }^{13}$ Lack of resources to refer patients for trauma specific help or lack of knowledge of resources and established links for referral, further limit medical staff's willingness to solicit disclosures or become involved..$^{414}$

Findings in the current study share other similarities with results of inquiries in other populations. For example, the size of the reported problem with women as victims in this study is 10 times higher than that among men. Other studies in health care settings also suggest that women sustain considerably higher rates of IPV related injury than men. ${ }^{15}{ }^{16}$ The low rates of injuries related to physical abuse among men make the cause of these injuries less amenable to screening detection.

The present investigation is large, covers all adult ages and both genders, and includes both rural and urban areas. Use, as a comparison group, of a large series of unintentional injuries is not a drawback because the differential etiological diagnosis of IPV injuries is generally oriented towards unintentional injuries (intentional injuries from persons other than intimate partners are in general freely attributed). Inclusion in the study of all injuries presented in emergency departments also allows the unbiased calculation of predictive values, which depend on the actual frequency of the phenomenon under investigation (in a case-control study with, say, equal number of IPV injuries and comparison, injuries predictive values cannot be calculated).

The main disadvantage is that attribution of injuries to IPV relies on reporting of events, which are believed to be frequently under-reported and may not even be documented in the medical record. Indeed, a recent Greek study has shown considerable under-reporting of suspected cases of child abuse in the medical files. ${ }^{17}$ A consequence of under-reporting is that actual frequencies of IPV injuries are probably higher than those found, although the odds ratio estimates should be little affected by this under-reporting. Another shortcoming of this study, which is, however, generic to this type of investigation, is that the findings may not be generalized in a straightforward way to populations living and functioning under very different conditions.

Identification of a health problem, estimation of its dimensions, and characterization of major risk factors are prerequisites for the launching of an effective preventive strategy. Although, probably underestimated, IPV injuries represent an important problem for Greeks as well as other populations and this investigation represents a first attempt to delineate the problem in this country or indeed, any other Mediterranean country.

More important, however, this study has helped identify injury characteristics, which have the properties required for the development of an effective screening tool towards the identification of IPV in emergency department settings. Actually, finding that only $1.1 \%$ of women in this study identified themselves as victims of IPV, lower than the lowest figure for women in studies in other countries, indicates the need for a more focused screening protocol to test whether the rate of IPV presentation in Greek accident and emergency settings is actually lower than rates in medical settings in other nations, or whether a more focused instrument would yield higher identification. Increasing public awareness of the occurrence of family violence in Greek society and discussions of assaults between partners in public discourse are also necessary to facilitate individual disclosures. Lastly, specialized training and development of basic awareness of the problem of family violence is critical to motivate the development of identification protocols and resources in all countries. Without an adequate resource network, health care professionals will remain reluctant to solicit disclosures from patients, and patients who do disclose will be frustrated or further endangered by the lack of services, once abuse is identified.

\section{ACKNOWLEDGEMENTS}

This research was partially supported by a grant from the European Commission [DG SANCO (2001 CVG3-315) SI325058] and from the Harvard Injury Control Research Center (partially funded by the US Centers for Disease Control and Prevention).

\section{Authors' affiliations}

E Petridou, Department of Hygiene and Epidemiology, Athens University Medical School, Athens, Greece and Department of Epidemiology, Harvard School of Public Health, Boston, USA

A Browne, E Lichter, Harvard Injury Control Research Center, Harvard University School of Public Health, Boston, USA

N Dessypris, X Dedoukou, D Alexe, Department of Hygiene and Epidemiology, Athens University Medical School, Athens, Greece

\section{REFERENCES}

1 Browne A. Violence against women: relevance for medical practitioners JAMA 1992;267:3148-89.

2 Abbott J, Johnson R, Koziol-McLain J, et al. Domestic violence against women. Incidence and prevalence in an emergency department population. JAMA 1995;273:1763-7.

3 Dearwater SR, Coben JH, Campbell JC, et al. Prevalence of intimate partner abuse in women treated at community hospital emergency departments. JAMA 1998;280:433-8.

4 Waalen J, Goodwin MM, Spitz AM, et al. Screening for intimate partner violence by health care providers. Barriers and interventions. Am J Prev Med 2000; 19:230-7

5 Dessypris N, Petridou E, Skalkidis Y, et al. Countrywide estimation of the burden of injuries in Greece: a limited resources approach. Accid Anal Prev (in press)

6 Coker AL, Smith PH, McKeown RE, et al. Frequency and correlates of intimate partner violence by type: physical, sexual, and psychological battering. Am J Public Health 2000;90:553-9.

7 Harwell TS, Spence MR. Population surveillance for physical violence among adult men and women, Montana 1998. Am J Prev Med $2000 ; 19: 321-4$

8 Plichta SB, Falik M. Prevalence of violence and its implications for women's health. Womens Health Issues 2001;11:244-58.

9 Sharps PW, Campbell J, Campbell D, et al. The role of alcohol use in intimate partner femicide. Am J Addict 2001;10:122-35.

10 Muelleman RL, Lenaghan PA, Pakieser RA. Battered women: injury locations and types. Ann Emerg Med 1996;28:486-92

11 Sugg NK, Inui T. Primary care physicians' response to domestic violence. Opening Pandora's box. JAMA 1992;267:3517-60.

12 Sugg NK, Thompson RS, Thompson DC, et al. Domestic violence and primary care. Attitudes, practices, and beliefs. Arch Fam Med 1999;8:301-6

13 Warshaw C. Domestic violence: challenges to medical practice. J Women's Health 1993;2:73-80.

14 Rodriguez MA, Sheldon WR, Baver HM, et al. The factors associated with disclosure of intimate partner abuse to clinicians. J Fam Pract 2001;50:338-44

15 Morse BJ. Beyond the Conflict Tactics Scale: assessing gender differences in partner violence. Violence Vict 1995;10:251-72.

16 Muelleman RL, Burgess P. Male victims of domestic violence and their history of perpetrating violence. Acad Emerg Med 1998;5:866-70.

17 Trogan I, Dessypris N, Moustaki M, et al. How common is abuse in Greece? Studying cases with femoral fractures. Arch Dis Child 2001;85:289-92. 